\title{
Elasticity Theory of a Twisted Stack of Plates
}

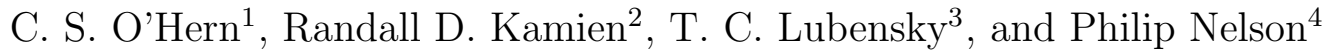 \\ Department of Physics and Astronomy, University of Pennsylvania \\ Philadelphia, PA 19104 \\ July 2, 1997
}

\begin{abstract}
We present an elastic model of B-form DNA as a stack of thin, rigid plates or base pairs that are not permitted to deform. The symmetry of DNA and the constraint of plate rigidity limit the number of bulk elastic constants contributing to a macroscopic elasticity theory of DNA to four. We derive an effective twist-stretch energy in terms of the macroscopic stretch $\epsilon$ along and relative excess twist $\sigma$ about the DNA molecular axis. In addition to the bulk stretch and twist moduli found previously, we obtain a twist-stretch modulus with the following remarkable properties: 1) it vanishes when the radius of the helical curve following the geometric center of each plate is zero, 2) it vanishes with the elastic constant $K_{23}$ that couples compression normal to the plates to a shear strain, if the plates are perpendicular to the molecular axis, and 3) it is nonzero if the plates are tilted relative to the molecular axis. This implies that a laminated helical structure carved out of an isotropic elastic medium will not twist in response to a stretching force, but an isotropic material will twist if it is bent into the shape of a helix.
\end{abstract}

PACS: 87.15.-v, 87.10.+e, 87.15.By.

\footnotetext{
${ }^{1}$ ohern@lubensky.physics.upenn.edu

${ }^{2}$ kamien@dept.physics.upenn.edu

${ }^{3}$ tom@lubensky.physics.upenn.edu

${ }^{4}$ nelson@dept.physics.upenn.edu
} 


\section{Introduction}

The elastic properties of DNA have become a focus of recent research [1, 2, 3, 4, 5, 6]. In particular, stretching experiments on single molecules of DNA now provide a direct probe of the bending, stretching, and twisting elasticity of DNA [7, 8, 10]. Understanding the elasticity of single molecules of DNA may be relevant in vivo; e.g., the recA protein which is responsible for homologous recombination in bacterial mitosis has been observed to stretch and twist DNA when it is bound to the DNA molecule 9].

Marko and Siggia recently modeled DNA as a thin, uniform rod with a linear bending elasticity and calculated the extension of the rod as a function of the applied stretching force 2]. This worm-like chain model was appropriate for stretching forces $f \ll \gamma$, where $\gamma \sim A k_{B} T / R^{2} \approx 200 \mathrm{pN}$ is the stretch modulus, $A \approx 50 \mathrm{~nm}$ is the bend persistence length, and $R=1 \mathrm{~nm}$ is the radius of the molecule. Forces in this regime pull on the thermal fluctuations of the molecular backbone but do not pull on the internal structure of DNA. The worm-like chain model accurately predicts the extension of the molecule to within $10 \%$ up to approximately $10 \mathrm{pN}[7$. Above $10 \mathrm{pN}[7$, 8 there are no longer thermal effects and DNA is stretched elastically. A strain variable $\epsilon$ must be introduced to describe increases in the molecular length after thermally induced contour length fluctuations are fully stretched [2, 11, 7, 8].

DNA is also characterized by the degree of twist of its phosphate backbones about the central axis. Relative twist excess or deficit from a state of preferred twist is parameterized by a signed twist variable $\sigma$. The chiral asymmetry of DNA allows for a twist-stretch coupling between $\sigma$ and $\epsilon$; this coupling is clearly visible in the recent experiments of Strick, et. al. [10, 4, 5]. Theoretical work has focused on calculating the extension of the molecule as function of both the applied stretching force and the applied excess twist [3, 6, 13]. However, in this paper we investigate a more microscopic origin of the twist-stretch coupling 14.

We introduce a simple elastic model of B-form DNA in which the molecule is viewed as a stack of thin, rigid plates that represent base pairs and are rotated and displaced relative to each other. (See Section 3 below for a review of the geometry of B-form DNA.) The centers of mass of the plates define a helical path around a straight central line, which we call the molecular axis. The perpendicular distance from the molecular axis to the helix is called the helix axis offset. Distortion energies in this model are controlled by 
an underlying continuum elastic energy with those elastic constants allowed by the anisotropic symmetry of DNA. Our model of DNA as a collection of thin, rigid plates has four contributing elastic constants coupling strains with spatial variation normal to the plates. Of particular importance is the elastic constant $K_{23}$ coupling compression perpendicular to the plates to a shear displacement parallel to the plates. $K_{23}$ vanishes if the phosphate backbones point in the same direction and also vanishes in the isotropic limit in which each plate has reflection and $\mathrm{C}_{4}$ or higher symmetries.

We calculate the twist-stretch coupling in terms of its elastic constants and the small helix axis offset. We find that the twist-stretch coupling has terms linear and quadratic in the helix axis offset. The coefficient of the linear term is proportional to the elastic constant $K_{23}$. In the isotropic limit or when $K_{23}$ vanishes, the twist-stretch coupling is quadratic in the helix axis offset as we calculated earlier [5, 12] in related but simpler models.

An important simplification of our model is the constraint that the plates comprising the DNA stack are rigid and undeformable. This is equivalent to setting to infinity all elastic constants coupling the strains within a plate. We thus neglect propeller, buckle, opening, etc. deformations 16 of individual base pairs and focus instead on inter-base pair deformations described by relative rotations (roll, tilt, and twist) and translations (slide, shift, and rise) of the base pairs 15, 16]. We believe this is a reasonable approximation for stretching forces in the range $1 \mathrm{pN}<f<10 \mathrm{pN}$ and relative twist excesses $\sigma<0.05$.

\section{Description of the Model}

DNA is composed of base pairs connected by two oppositely-directed sugarphosphate backbones that wrap in two helices around the central molecular axis to produce the major and minor grooves. A cross-section of DNA normal to the phosphate backbones is shown in Fig.1. To construct our model for DNA, we first imagine unwinding the helix so that the phosphate backbones describe two straight, parallel paths. This unwound structure is an elastic rod characterized by an elastic constant tensor $K_{i j k l}$ with components constrained by the symmetry of the rod. Each cross-section of the rod looks like Fig.

\footnotetext{
${ }^{5}$ Each phosphate backbone has a particular orientation: either 3'-5' or 5'-3'.
} 
1. Let the $z$-axis be parallel to the phosphate backbones, and let the $x$ axis pass through the midpoint and perpendicular to the line connecting the backbones. The only symmetry operation of this rod is a rotation through $\pi$ about the $x$-axis that causes the axes to transform as $x \rightarrow x, y \rightarrow-y$, and $z \rightarrow-z$. This symmetry allows for a maximum of 13 independent elastic constants. Our assumption of the rigid shape of each cross-section in the $x-y$ plane eliminates from consideration all strains that require spatial derivatives with respect to $x$ or $y$. Thus we need only consider the strains $u_{z z}, u_{z x}$, and $u_{z y}$ and the four elastic constants $K_{33}=K_{z z z z}, K_{11}=K_{z x z x} / 4$, $K_{22}=K_{z y z y} / 4$, and $K_{23}=K_{z y z z} / 2$ permitted by symmetry that couple these strains. The elastic constant $K_{23}$ is allowed because the two phosphate backbones are oppositely-directed. If they were undirected or pointed in the same direction, the reflection $y \rightarrow-y$ would be a symmetry and $K_{23}$ would be zero. If, in addition, a rotation by $\pi / 2$, which transforms $x \rightarrow y$ and $y \rightarrow-x$, were a symmetry, there would be only two elastic constants $K_{33}$ and $K=K_{11}=K_{22}$. We will refer to this case with two elastic constants as the isotropic limit. We view the cross-sectional slices as rigid plates that are coupled elastically via the elastic constants $K_{33}, K_{11}, K_{22}$, and $K_{23}$.

We now imagine that chiral forces inherent to DNA distort the straight rod to a helical structure in which the centers of mass of the rigid plates describe a helical path about a straight helical axis. This helical structure can be produced by displacing and rotating neighboring plates by a constant amount. We assume that chiral energies leading to a ground-state helical structure are linear in strain (i.e surface terms) so that the energies of distortions from the ground state are determined by the elasticity of the original untwisted rod, i.e., by the elastic constants $K_{33}, K_{11}, K_{22}$, and $K_{23}$. In the following sections, we will derive the twist-stretch coupling in terms of these constants and the helix axis offset.

\section{Geometry of B-Form DNA}

We now consider three different ways of stacking our thin, rigid plates to create a helical model of the straight state of B-DNA. We first imagine simply twisting the plates about the long axis passing through the center of mass of the original rod to create shape I. In this shape, the geometric centers

of each slice lie on top of one another, the slices are rotated about the long 
axis relative to one another, and the slices remain perpendicular to the long axis. Shape II is obtained by first twisting the plates about the long axis and then removing material from the side of each plate opposite to the phosphate backbones. The line connecting the new geometric centers of each slice follows a helix. The slices remain perpendicular to the helix axis about which the geometric centers of each plate rotate. (In the discussion below we use the terms helix axis and DNA molecular axis interchangeably.) Shape III is obtained by bending the long axis of the sliced rod into a helix. The curve $\vec{r}$ connecting the geometric centers of each plate again follows a helix, but now the plates are perpendicular to the local tangent to $\vec{r}$ instead of the helix axis.

We note that in the unstressed state of B-form DNA the plates are roughly perpendicular to the molecular axis, and their geometric centers nearly coincide with the molecular axis[17]. Thus to study small deformations of B-DNA, it suffices to restrict our attention to the case where shapes II and III are small perturbations of shape I. In shape II, the amount of material removed from each slice and hence the radius of the helix is small and in shape III the bend of the rod axis away from the molecular axis is also small. Shapes II and III can be described as twisted stacks of plates with small helix axis offsets. If the collection of plates has a helix axis offset, the plates are not stacked with one directly on top of the other; instead, the shift vector has a component perpendicular to the molecular axis. We will see that in our model the helix axis offset is the origin of the twist-stretch coupling.

\section{Elasticity Theory}

In what follows we will develop a long-wavelength elasticity theory for a collection of stacked, rigid plates in terms of small deviations of the shifts and rotations away from their unstressed values. We will then eliminate the shift and rotation variables in favor of the stretch along the molecular axis $\epsilon$ and the relative excess twist about the molecular axis $\sigma$. This will allow us to find the bulk twist and stretch moduli in terms of four elastic constants and geometric properties of the molecule. More importantly, it will also enable us to calculate the twist-stretch coupling and determine how it scales with the small helix axis offset.

As shown in Fig. 1 we inscribe on each plate a right-handed, orthonormal 
triad $\mathbf{e}_{\alpha}(n)$ where $\alpha=1,2,3$ and $n$ is a unitless parameter which labels the plate. In the following, Roman indices run over Cartesian space coordinates $(x, y, z)$ and Greek indices label the plate-fixed coordinate system $(1,2,3)$. $\mathbf{e}_{1}$ and $\mathbf{e}_{2}$ lie in the plane of the plate, and $\mathbf{e}_{3}$ is perpendicular to the plate. The coordinates on each plate are labeled by the pair $\left(\eta_{1}, \eta_{2}\right)$ which corresponds to the point on the plate $\eta_{1} \mathbf{e}_{1}(n)+\eta_{2} \mathbf{e}_{2}(n)$. We must also specify the origin on each plate. We choose the origin to be the geometric center (i.e., center of mass) of the plate, but this choice is arbitrary and does not affect the elasticity theory, as we will discuss in the Appendix. We can now describe the trajectory in space of the plates and the associated plate-fixed triads. We allow two sets of parameters: a vector of rotation rates $\vec{\omega}$ and shifts $\vec{\delta}$. We decompose $\vec{\omega}$ and $\vec{\delta}$ in the plate-fixed basis $\mathbf{e}_{\alpha}(n)$ and assume that the components $\omega_{\alpha}$ and $\delta_{\alpha}$ are constants independent of $n$.

$$
\begin{aligned}
& \vec{\omega}=\omega_{\alpha} \mathbf{e}_{\alpha}(n) \\
& \vec{\delta}=\delta_{\alpha} \mathbf{e}_{\alpha}(n), \quad \text { where } \quad \alpha=1,2,3
\end{aligned}
$$

The vector of rotation rates $\vec{\omega}$ describes the rate of change of the orientation of neighboring plates, and thus

$$
\frac{d \mathbf{e}_{\alpha}}{d n}=\vec{\omega} \times \mathbf{e}_{\alpha}=-\epsilon_{\alpha \beta \gamma} \omega_{\beta} \mathbf{e}_{\gamma},
$$

where $\epsilon_{\alpha \beta \gamma}$ is the antisymmetric tensor. The second set of parameters $\vec{\delta}$ describes the relative displacement of two neighboring plates. We choose $\vec{\delta}$ to be the relative displacement of the origin $\vec{r}(n)$ of the $\left(\eta_{1}, \eta_{2}\right)$ coordinate system. We have

$$
\frac{d \vec{r}(n)}{d n}=\vec{\delta}=\mathbf{e}_{\alpha} \delta_{\alpha}
$$

Note that according to our definitions $\vec{\omega}$ is unitless and $\vec{\delta}$ has units of length. Finally, the position in space of the point $\left(\eta_{1}, \eta_{2}\right)$ on plate $n$ is simply $\vec{x}\left(\eta_{1}, \eta_{2}, n\right)=\vec{r}(n)+\eta_{1} \mathbf{e}_{1}(n)+\eta_{2} \mathbf{e}_{2}(n)$. For constant $\omega_{\alpha}$ and $\delta_{\alpha}$, these equations will, in general, describe a helical structure. We may solve (2) and (3) to find:

$\vec{r}(n)=\mathbf{e}_{\beta}(0)\left\{n \frac{\omega_{\beta}(\vec{\delta} \cdot \vec{\omega})}{\omega^{2}}+\left(\delta_{\beta}-\omega_{\beta} \frac{(\vec{\delta} \cdot \vec{\omega})}{\omega^{2}}\right) \frac{\sin (|\vec{\omega}| n)}{|\vec{\omega}|}+\frac{\epsilon_{\beta \alpha \gamma} \delta_{\alpha} \omega_{\gamma}}{\omega^{2}} \cos (|\vec{\omega}| n)\right\}$, 
Table 1: Geometric Properties of Several Helices

\begin{tabular}{ccccc} 
Shape & $\vec{\delta}$ & $\vec{\omega}$ & Helix Axis Offset & Pitch $/ 2 \pi$ \\
\hline I & $\left(0,0, \delta_{3}\right)$ & $\left(0,0, \omega_{3}\right)$ & 0 & $\delta_{3} / \omega_{3}$ \\
II & $\left(0, \delta_{2}, \delta_{3}\right)$ & $\left(0,0, \omega_{3}\right)$ & $\delta_{2}$ & $\delta_{3} / \omega_{3}$ \\
III & $\left(0,0, \delta_{3}\right)$ & $\left(0, \omega_{2}, \omega_{3}\right)$ & $\omega_{2} \delta_{3} / \sqrt{\omega_{2}^{2}+\omega_{3}^{2}}$ & $\delta_{3} \omega_{3} /\left(\omega_{2}^{2}+\omega_{3}^{2}\right)$
\end{tabular}

with $\omega^{2}=\omega_{\alpha} \omega_{\alpha}$. Examination of (四) shows that the geometric center moves on average in the direction $\mathbf{e}_{\beta}(0) \omega_{\beta} /|\vec{\omega}|$ and traces out a helix with helix axis offset $r=|\vec{\omega} \times \vec{\delta}| /|\vec{\omega}|$ and pitch $p=2 \pi \vec{\delta} \cdot \vec{\omega} / \omega^{2}$. The shift vector, rotation vector, helix axis offset, and pitch are listed in Table 1 for each of the three shapes of B-form DNA we are considering.

In a deformed state, the displacement and rotation rates change to $\vec{\delta}^{\prime}=$ $\vec{\delta}+\vec{\Delta}(n)$ and $\vec{\omega}^{\prime}=\vec{\omega}+\vec{\Omega}(n)$ and define new positions $\vec{x}^{\prime}\left(\eta_{1}, \eta_{2}, n\right)$ for points on plate $n$. Our goal is to calculate the energy of this deformed state relative to the equilibrium helical state to second order in the small parameters $\vec{\Delta}$ and $\vec{\Omega}$, which can in general depend on $n$. We consider plates of thickness $\delta n$ and calculate the energy in the deformed state of each plate to order $\delta n$, ignoring terms of order $(\delta n)^{2}$ or higher. We then sum over all plates to obtain the total energy of the rod. Since the energy of each plate is proportional to $\delta n$, the sum over all plates can be converted into an integral over $n$.

The energy of the $n$th plate will depend on the values of $\vec{\Delta}$ and $\vec{\Omega}$ at $n$ and, to the order we consider, not on their derivatives with respect to $n$. The energy of each plate will be the same function of $\vec{\Delta}$ and $\vec{\Omega}$ for every $n$, so we need only calculate the energy of a single reference plate. Our elastic theory provides us with distortion energies of the reference plate as a function of the strains $u_{i j}=\frac{1}{2}\left(\partial_{i} u_{j}+\partial_{j} u_{i}\right)(i, j=x, y, z)$, where $\vec{u}=\vec{x}^{\prime}-\vec{x}$ is the displacement variable and $x, y$, and $z$ are the Cartesian coordinates of the reference plate. By a suitable choice of orientation we may take $\mathbf{e}_{1}=\hat{x}$, $\mathbf{e}_{2}=\hat{y}$, and $\mathbf{e}_{3}=\hat{z}$ for the plate of interest. The free energy for a single plate with thickness $\delta z$ is (in units of $k_{B} T$ ):

$$
\frac{F_{\text {plate }}}{k_{B} T}=\frac{1}{2} \int_{z}^{z+\delta z} \int d x d y d z K_{i j k l} u_{i j} u_{k l} .
$$


Our goal is to express this energy as a function of $\vec{\Delta}$ and $\vec{\Omega}$ and change integration variables from $x, y$, and $z$ to $\eta_{1}, \eta_{2}$, and $n$. The Jacobian relating the two coordinate systems is found by taking $\eta_{\alpha}$ and $n$ derivatives of $\vec{x}\left(\eta_{1}, \eta_{2}, n\right)$ and remembering that $\mathbf{e}_{\alpha} \cdot \mathbf{e}_{\beta}=\delta_{\alpha \beta}$.

$$
d x d y d z=\left|\delta_{3}+\epsilon_{3 \beta \gamma} \omega_{\beta} \eta_{\gamma}\right| d \eta_{1} d \eta_{2} d n .
$$

To calculate the strain tensor $u_{i j}$ we must take the derivatives of $\vec{u}\left(\eta_{1}, \eta_{2}, n\right)$ with respect to $\eta_{\alpha}$ and $n$ and then relate these coordinates to Cartesian coordinates $x, y$, and $z$ fixed on the plate. Since the derivatives of $u_{i}$ with respect to $\eta_{\alpha}$ only include terms proportional to $\delta n$, the only derivatives in the strain tensor contributing to the total free energy in (5) are those in the $n$-direction. We therefore only need to calculate $d \vec{u} / d n$. Using the equations of motion, (2) and (3), and the expression for $\vec{x}\left(\eta_{1}, \eta_{2}, n\right)$, we have to linear order in $\Omega_{\alpha}$ and $\Delta_{\alpha}$

$$
\frac{d \vec{u}\left(\eta_{1}, \eta_{2}, n\right)}{d n}=\mathbf{e}_{\alpha}\left[\Delta_{\alpha}+\epsilon_{\alpha \beta \gamma} \Omega_{\beta} \eta_{\gamma}\right]+\left(\delta_{\alpha}+\epsilon_{\alpha \beta \gamma} \omega_{\beta} \eta_{\gamma}\right)\left[\mathbf{e}_{\alpha}^{\prime}-\mathbf{e}_{\alpha}\right]
$$

We can neglect the last terms in (7) since they also are proportional to $\delta n$. (We note that deformations of the base pairs can be included if we retain these $\delta n$ contributions.)

We choose the slab at $n$ to have its internal triad point along a Cartesian coordinate system $(x, y, z)$ fixed on the plate with the $z$-direction aligned with $\mathbf{e}_{3}$ and the $x$ - and $y$-directions aligned with $\mathbf{e}_{1}$ and $\mathbf{e}_{2}$ respectively. To leading order in $\Omega_{\alpha}$ and $\Delta_{\alpha}$, the only relevant components of the strain tensor come from the derivatives

$$
\partial_{z} u_{i}=\frac{\partial u_{i}}{\partial n} \frac{\partial n}{\partial z}=\left(\Delta_{i}+\epsilon_{i j k} \Omega_{j} \eta_{k}\right) / \delta_{3}
$$

where $\partial n / \partial z=1 / \delta_{3}$ for the three shapes we are considering. In (8) the sums over $\alpha, \beta$, and $\gamma$ have been replaced by sums over $i, j$, and $k$ because we chose the body-fixed coordinates to be along $(x, y, z)$. We have now reduced the number of effective elastic constants from 13 to 4 since only $z$ derivatives contribute to the free energy. The free energy of a single plate in terms of the strains $\vec{\Delta}$ and $\vec{\Omega}$ and the coordinates $\eta_{1}, \eta_{2}$, and $n$ is:

$\frac{F_{\text {plate }}}{k_{B} T}=\int_{n}^{n+\delta n} d n \int d^{2} \eta \frac{\left|\delta_{3}+\epsilon_{3 \beta \gamma} \omega_{\beta} \eta_{\gamma}\right|}{2 \delta_{3}^{2}}\left\{K_{i j}\left(\Delta_{i}+\epsilon_{i l m} \Omega_{l} \eta_{m}\right)\left(\Delta_{j}+\epsilon_{j k n} \Omega_{k} \eta_{n}\right)\right\}$, 
where $K_{i j}$ is related to $K_{i j k l}$ by

$$
K_{i j}= \begin{cases}\frac{1}{4} K_{z i z j} & i=j=x \text { or } i=j=y \\ \frac{1}{2} K_{z i z j} & i=y, j=z \\ K_{z i z j} & i=j=z\end{cases}
$$

We see that our elasticity theory of DNA as a stack of thin, rigid plates has four contributing elastic constants, $K_{x x}, K_{y y}, K_{y z}$, and $K_{z z}$. Below we will refer to these elastic constants as $K_{11}, K_{22}, K_{23}$, and $K_{33}$, respectively, since the plate-fixed axes rotate in space as we move along the helical stack. We note that an isotropic theory has only two contributing constants, $K=$ $K_{11} / 2=K_{22} / 2$ and $K_{33}$. The total free energy for the collection of plates is obtained by adding up the contributions to the free energy from each plate. The total free energy will have the same form as (9) with the Cartesian indices on the strains $(x, y, z)$ replaced by the plate-fixed indices $(1,2,3)$.

\section{DNA Effective Free Energy}

We now sum up the contributions from all slices and do the integrals over $\eta_{1}$ and $\eta_{2}$ in (9) to get an effective theory for the DNA molecule. Before we actually do this, we will make some further simplifications and approximations. We first use the fact that under the DNA symmetry operation $\eta_{2}$ switches sign and hence all moments odd in $\eta_{2}$ are zero: $\left\langle\eta_{2}\right\rangle=\left\langle\eta_{1}^{m} \eta_{2}\right\rangle=0$ $(m=1,2,3, \ldots)$ where $\langle\cdot\rangle \equiv \mathcal{A}^{-1} \int d^{2} \eta(\cdot)$ and $\mathcal{A}$ is the cross sectional area in the $\eta_{1}-\eta_{2}$ plane. We also locate the origin of each plate at its geometric center so that $\left\langle\eta_{1}\right\rangle=0$.

In the unstressed configuration of B-form DNA the base pairs make an angle of close to $\pi / 2$ with the molecular axis 17]. We therefore take $\omega_{1,2} l_{0} \ll$ $\delta_{3}$ where $\delta_{3} \approx 3.4 \AA$ is the axial rise of the base pairs. In addition, the geometric centers of the plates are roughly located on the molecular axis [17]; we therefore also assume $\delta_{1,2} / \delta_{3} \ll 1$. We will characterize the unstressed straight state of B-DNA by two known macroscopic parameters (the axial rise $\delta_{3}$ and the twist rate of the plates about the molecular axis $\omega_{3}=2 \pi \delta_{3} / l_{0} \approx$ $0.63 \mathrm{rad})$ and two unknown microscopic parameters $\left(\delta_{2}\right.$ and $\left.\omega_{2}\right)$. In what

follows we will assume that $\delta_{2} / \delta_{3}, \omega_{2} / \omega_{3}$, and the second moments $\left\langle\eta_{1}^{2}\right\rangle / l_{0}^{2}$ and $\left\langle\eta_{2}^{2}\right\rangle / l_{0}^{2}$ are small and work to quadratic order in these quantities. 
The effective free energy is given below in units of $k_{B} T$. We neglect the $\omega_{2}$ contribution arising from the Jacobian in (9) since it yields terms that are third order in $\omega_{2} / \omega_{3},\left\langle\eta_{1}^{2}\right\rangle / l_{0}^{2}$, and $\left\langle\eta_{2}^{2}\right\rangle / l_{0}^{2}$. Including these terms does not alter our findings below for the twist-stretch coupling:

$$
\begin{array}{r}
\frac{F_{\mathrm{DNA}}}{k_{B} T}=\left(\mathcal{A} / 2 \delta_{3}\right) \int d n\left\{K_{11} \Delta_{1}^{2}+K_{22} \Delta_{2}^{2}+K_{33} \Delta_{3}^{2}+K_{33}\left\langle\eta_{2}^{2}\right\rangle \Omega_{1}^{2}+\right. \\
\left.K_{33}\left\langle\eta_{1}^{2}\right\rangle \Omega_{2}^{2}+\left(K_{11}\left\langle\eta_{2}^{2}\right\rangle+K_{22}\left\langle\eta_{1}^{2}\right\rangle\right) \Omega_{3}^{2}-2 K_{23}\left\langle\eta_{1}^{2}\right\rangle \Omega_{2} \Omega_{3}+2 K_{23} \Delta_{2} \Delta_{3}\right\} .
\end{array}
$$

We note from (11) that the elastic constant $K_{33}$ acts as a stretch modulus and a bend modulus.

\section{Twist-Stretch Coupling}

Motivated by recent stretching experiments on torsionally constrained single molecules of DNA 10, we rewrite (11) in terms of the relative overtwist $\sigma$ about the molecular axis and the relative stretch $\epsilon$ along the molecular axis. To accomplish this we must first find $\epsilon$ and $\sigma$ in terms of the rotation and shift variables $\Omega_{i}$ and $\Delta_{i}$. After we incorporate $\epsilon$ and $\sigma$ into (11), we minimize over the remaining unconstrained variables. This gives us the twist-stretch energy in terms of the elastic constants $K_{i j}$ and the geometric parameters of the DNA helix. We can then identify the stretch modulus $B$, the twist modulus $C$, and the twist-stretch modulus $D$. We note that this twiststretch energy neglects the effects of thermal fluctuations; these effects have been studied recently 6, 13.

We now derive the twist-stretch coupling for the three shapes shown in Table 1 by considering the general case $\omega_{2}, \delta_{2} \neq 0$ and then neglecting $\omega_{2} \delta_{2}$ cross terms. The unstressed configuration is therefore $\vec{\omega}=\left(0, \omega_{2}, \omega_{3}\right)$ and $\vec{\delta}=\left(0, \delta_{2}, \delta_{3}\right)$. Each of the three equilibrium shapes mentioned previously can be obtained from this parameterization, for instance, shape II is generated by taking $\delta_{2} \neq 0$ and $\omega_{2}=0$. We define the stretch along the molecular axis as the relative deviation in the extension $L$ along the molecular axis from its unstressed value $L_{0}$,

$$
\epsilon=\frac{L}{L_{0}}-1
$$

To find $\epsilon$ in terms of $\Delta_{i}$ and $\Omega_{i}$ we must determine the extension $L$ from (田). We see that each base pair step increases the extension by an amount 
$\vec{\delta} \cdot \vec{\omega} /|\vec{\omega}|$ in the direction $\vec{\omega} /|\vec{\omega}|$. The total extension for $N$ steps in the distorted configuration is therefore

$$
L=N \vec{\delta}^{\prime} \cdot \frac{\vec{\omega}^{\prime}}{\left|\vec{\omega}^{\prime}\right|}
$$

where the primes refer to the distorted state, i.e. $\overrightarrow{\delta^{\prime}}=\left(\Delta_{1}, \delta_{2}+\Delta_{2}, \delta_{3}+\Delta_{3}\right)$. We can now expand (12) to linear order in the $\Omega_{i}$ and $\Delta_{i}$ and to quadratic order in the small parameters $\delta_{2} / \delta_{3}$ and $\omega_{2} / \omega_{3}$ :

$$
\epsilon=\frac{\Delta_{3}}{\delta_{3}}+\left(\frac{\delta_{2}}{\delta_{3}}-\frac{\omega_{2}}{\omega_{3}}\right) \frac{\Omega_{2}}{\omega_{3}}+\frac{\omega_{2}}{\omega_{3}} \frac{\Delta_{2}}{\delta_{3}}+\frac{\omega_{2}^{2}}{\omega_{3}^{2}} \frac{\Omega_{3}}{\omega_{3}} .
$$

Note that when the helix axis offset of the helical stack is zero $\left(\delta_{2}=\omega_{2}=0\right)$, the stretch reduces to $\epsilon=\Delta_{3} / \delta_{3}$. We also note that when $\omega_{2}=0 \Delta_{3}$ and $\Omega_{2}$ are the only distortions contributing to the stretch.

We must express the relative excess link $\sigma=\left(L k / L k_{0}\right)-1$ in terms of the $\Omega_{i}$, where the linking number $L k$ is the number of times the plates rotate about the molecular axis over the length of the stack. In its unstressed state, DNA has linking number $L k_{0}=L_{0} / l_{0}$. (We are working in the force regime where the molecular axis is nearly straight, and hence link and relative twist about the molecular axis are synonymous.) We see from (4) that it takes $2 \pi /|\vec{\omega}|$ steps to complete one revolution about the molecular axis. Therefore in $N$ steps the top plate has rotated by $N|\vec{\omega}| / 2 \pi$ relative to the bottom plate. The relative excess twist about the molecular axis is therefore

$$
\sigma=\frac{\left|\vec{\omega}^{\prime}\right|}{|\vec{\omega}|}-1,
$$

where $\vec{\omega}^{\prime}=\left(\Omega_{1}, \omega_{2}+\Omega_{2}, \omega_{3}+\Omega_{3}\right)$. Expanding (15) to linear order in the $\Omega_{i}$ and to quadratic order in $\omega_{2} / \omega_{3}$, we find

$$
\sigma=\frac{\omega_{2}}{\omega_{3}} \frac{\Omega_{2}}{\omega_{3}}+\left(1-\frac{\omega_{2}^{2}}{\omega_{3}^{2}}\right) \frac{\Omega_{3}}{\omega_{3}} .
$$

Note that when $\omega_{2}=0$, the base pairs are perpendicular to the molecular axis and the relative excess twist is simply $\Omega_{3} / \omega_{3}$.

Solving (14) for $\Delta_{3}$ and (16) for $\Omega_{3}$, we substitute into (11) and minimize over $\Delta_{1,2}$ and $\Omega_{1,2}$. This gives the effective twist-stretch energy per length 
along the molecular axis:

$$
\frac{F_{T S}(\epsilon, \sigma)}{N \delta_{3} k_{B} T}=\frac{\mathcal{A}}{2}\left(B \epsilon^{2}+C \sigma^{2}+2 D \epsilon \sigma\right)
$$

where $N$ is the total number of plates, $B$ is the stretch modulus, $C$ is the twist modulus, and $D$ is the twist-stretch modulus. To zeroth order in the small parameters $\delta_{2} / \delta_{3}$ and $\omega_{2} / \omega_{3}$ the stretch and twist moduli are:

$$
\begin{aligned}
& B=K_{33}-K_{23}^{2} / K_{22} \\
& C=\left(\omega_{3}^{2} / \delta_{3}^{2}\right)\left(K_{11}\left\langle\eta_{2}^{2}\right\rangle+\left\langle\eta_{1}^{2}\right\rangle\left(K_{22}-K_{23}^{2} / K_{33}\right)\right) .
\end{aligned}
$$

$B, C$, and $D$ have dimension $L^{-3}$ and therefore the coefficients of the quadratic dimensionless strains in (17) scale as a persistence length divided by the square of the helical pitch as found previously[5]. The twist-stretch couplings $D_{i}$ for the three shapes given in Table 10 are shown below to lowest order in the helix axis offsets $r_{i}$ :

$$
\begin{aligned}
D_{\mathrm{I}} & =0 \\
D_{\mathrm{II}} & \approx \frac{K_{23}}{K_{33}}\left(K_{33}-\frac{K_{23}^{2}}{K_{22}}\right) \frac{r_{\mathrm{II}}}{\delta_{3}} \\
D_{\mathrm{III}} & \approx \frac{K_{23}}{K_{33}}\left(K_{33}-\frac{K_{23}^{2}}{K_{22}}\right) \frac{r_{\mathrm{III}}}{\delta_{3}}+\left(K_{11} \frac{\left\langle\eta_{2}^{2}\right\rangle}{\left\langle\eta_{1}^{2}\right\rangle}+K_{22}-K_{33}\right)\left(\frac{r_{\mathrm{III}}}{\delta_{3}}\right)^{2},
\end{aligned}
$$

where the helix axis offsets for each shape are $r_{\mathrm{I}}=0, r_{\mathrm{II}}=\delta_{2}$, and $r_{\mathrm{III}} \approx$ $\omega_{2} \delta_{3} / \omega_{3}$. In performing the calculation of the twist-stretch moduli we have made two simplifications: 1) we have dropped the quadratic terms in the helix axis offset that are proportional to $K_{23}$ and 2) we have not included the $\omega_{2}$ contributions arising from the Jacobian in (9). This last simplification does not affect our conclusions concerning the twist-stretch coupling (TS) shown in Table 2 since $\omega_{2}$ contributions can only affect the twist-stretch coupling of shape III and shape III already has twist-stretch couplings in both the isotropic and anisotropic models.

We see from (19) that the twist-stretch coupling in our rigid plate model vanishes when the helix axis offset $r$ vanishes. However, calculations for a pretwisted rod of material with a three-dimensional isotropic elasticity yield a twist-stretch coupling for zero helix axis offset provided the rod crosssection is not circular 18]. To achieve consistency with our results, it seems 
Table 2: Determination of the twist-stretch coupling for each shape of Bform DNA is shown below. The symbol TS signifies that there is a nonzero twist-stretch coupling.

\begin{tabular}{cccc} 
Shape & $\begin{array}{c}\text { Helix Axis } \\
\text { Offset }\end{array}$ & $\begin{array}{c}\text { Isotropic Model } \\
\left(K_{23}=0\right)\end{array}$ & $\begin{array}{c}\text { Anisotropic Model } \\
\left(K_{23} \neq 0\right)\end{array}$ \\
\hline I & 0 & 0 & 0 \\
II & $\delta_{2}$ & 0 & TS \\
III & $\omega_{2} \delta_{3} / \omega_{3}$ & TS & TS
\end{tabular}

likely that the magnitude of this coupling decreases as the elastic constants coupling in-plane strains $\left(i . e ., u_{x x}, u_{x y}, u_{y y}\right)$ increase. We can thus view our calculation as the limit when these elastic constants diverge.

We also see that in the isotropic limit $\left(K_{23}=0\right)$ the twist-stretch coupling for shape III scales quadratically with the small helix axis offset as found previously in Refs. [5, 12]. However, when the $K_{23}$ anisotropy is nonzero, the twist-stretch coupling scales linearly with the small helix axis offset. We note that the twist-stretch coupling in the isotropic limit vanishes for shape II since it has $\omega_{2}=0$. However, shape III yields a twist-stretch coupling in both the isotropic and anisotropic cases since it has $\omega_{2} \neq 0$. This may be explained by considering the manner in which the plates are stacked in the two different geometries. In shape II the plates are stacked perpendicular to the molecular axis. In this case, the plates can move along the molecular axis without rotating in response to the stretching force. In shape III the plates are not perpendicular to the molecular axis, but are instead perpendicular to the local tangent $d \vec{r} / d n$. In order to align the plates perpendicular to the molecular axis, they must twist. This implies that an isotropic rod bent into the shape of a helix will have a twist-stretch coupling, but carving a helical shape out of isotropic material will not produce a twist-stretch coupling. 


\section{Comparison to the Ribbon Model of DNA}

We compare our plate model to a ribbon model of DNA recently discussed in Ref. [12]. There we modeled DNA as a thin helical ribbon that is only allowed to stretch along the ribbon axis. Changes in extension along the molecular axis arose from changes in the angle the ribbon tangent makes with the molecular axis and from stretching the ribbon axis. We showed that the twist-stretch coupling scaled quadratically with the helix axis offset if the twist-bend, twist-stretch, and bend-stretch couplings for twists and bends about and stretches along the ribbon axis were set to zero.

To make a direct comparison between the two models we consider the unstressed configuration $\vec{\delta}=\left(0,0, \delta_{3}\right)$ and $\vec{\omega}=\left(0, \omega_{2}, \omega_{3}\right)$ and do not allow stretch along the 1 and 2 axes of each plate by setting $\Delta_{1}=\Delta_{2}=0$. This corresponds to a helical stack of plates with the planes of the plates perpendicular to the tangent to the curve $\vec{r}$ connecting the geometric centers of the plates and stretching only allowed along the tangent $d \vec{r} / d n$. When we set $\Delta_{1}=\Delta_{2}=0$ in (11), we find a free energy identical to the one studied previously [12]. We see below that our expression for the free energy admits all of the couplings found in the ribbon model, namely the bend-stretch $\left(\Omega_{2} \Delta_{3}\right)$, twist-bend $\left(\Omega_{2} \Omega_{3}\right)$, and twist-stretch $\left(\Omega_{3} \Delta_{3}\right)$ couplings. (We have included the $\omega_{2}$ contributions from the Jacobian and kept each coupling to lowest order in $\omega_{2} / \omega_{3}$ and the second moments.)

$$
\begin{aligned}
& \frac{F_{\text {ribbon }}}{k_{B} T}= \\
& \quad\left(\mathcal{A} / 2 \delta_{3}\right) \int d n\left\{K_{33}\left\langle\eta_{2}^{2}\right\rangle \Omega_{1}^{2}+K_{33}\left\langle\eta_{1}^{2}\right\rangle \Omega_{2}^{2}+\left(K_{11}\left\langle\eta_{2}^{2}\right\rangle+K_{22}\left\langle\eta_{1}^{2}\right\rangle\right) \Omega_{3}^{2}+K_{33} \Delta_{3}^{2}+\right. \\
& \left.2 K_{33} \frac{\omega_{2}}{\delta_{3}}\left\langle\eta_{1}^{2}\right\rangle \Omega_{2} \Delta_{3}-2 K_{23}\left\langle\eta_{1}^{2}\right\rangle \Omega_{2} \Omega_{3}-2 K_{23} \frac{\omega_{2}}{\delta_{3}}\left\langle\eta_{1}^{2}\right\rangle \Omega_{3} \Delta_{3}\right\} .
\end{aligned}
$$

We find that the presence of the the last two terms leads to twist-stretch couplings that scale linearly with the helix axis offset $D \sim\left(K_{23} / K_{33}\right) r_{\mathrm{III}} / \delta_{3}$. If we set $K_{23}=0$, the lowest order contribution is quadratic in the helix axis offset and we obtain the result $D \sim K_{33}\left(r_{\mathrm{III}} / \delta_{3}\right)^{2}$ found previously [5, 12]. 


\section{Conclusion}

We have presented an elasticity theory for B-form DNA modeled as a stack of thin, rigid plates. The symmetry of the DNA molecule and the assumption of plate rigidity enabled us to reduce the number of contributing elastic constants to four. We then derived an effective twist-stretch energy for DNA in terms of the relative excess twist $\sigma$ about the molecular axis and the stretch $\epsilon$ along the molecular axis. We found an effective twist-stretch coupling with terms that scale linearly and quadratically with the small helix axis offset. We have argued that deformations of the base pairs are more costly than deviations in the shifts and rotations of the base pairs from their unstressed values. We therefore conclude that in the force regime where deformations of the plates are negligible, the twist-stretch coupling is due to a nonzero helix axis offset. We have also found that shape II, in which the normals to the base pairs parallel to the molecular axis, does not have a twist-stretch coupling in the limit $K_{23}=0$. Since recent experiments show that there is a large twist response to stretch, this implies that either the coupling $K_{23}$ is relevant for an elastic description of B-DNA or that the unstressed straight state of B-DNA is composed of base pairs that tilt relative to the molecular axis.

An important next step is to estimate the elastic constant $K_{23}$ to determine the relative magnitudes of the isotropic and anisotropic terms in the twist-stretch coupling. One might also calculate the twist-stretch coupling for DNA plasmids where the unstressed state of the molecular backbone is circular rather than linear. This calculation could provide a theoretical estimate of the twist-stretch coupling found in experiments on complexes of Rec-A protein with DNA plasmid [9].

\section{Acknowledgments}

We would like to thank D. Bensimon, J.F. Marko, and J.D. Moroz for helpful comments. CSO, RDK, and TCL were supported in part by NSF grant DMR94-23114. RDK was also supported in part by NSF grant DMR9520175 and PN was partially supported by NSF grant DMR95-07366. 


\section{Appendix: Translational Invariance of the Plate Origin}

We comment here on the invariance of our model under the choice of the plate origin. This is an important feature since a twisted stack of plates can be described by an infinite number of one-dimensional curves depending on the choice of the reference point on each plate. We proceed by considering a change in the origin, or, in particular a change in the coordinate system such that $\vec{x}=\left(\eta_{1}, \eta_{2}, s\right) \rightarrow \tilde{x}=\left(\eta_{1}, \eta_{2}, s\right)+\vec{R}$ where $\vec{R}$ is an arbitrary vector. While this translation does not affect the relative rotation of two consecutive plates $\vec{\omega}$, it does affect the relative translation. Consider the action of $g=(\vec{\omega}, \vec{\delta})$ on $\vec{x}$ :

$$
\vec{x} \stackrel{g}{\longrightarrow} \vec{x}+\vec{\delta}-\vec{\omega} \times \vec{x} .
$$

We now consider the transformation in (21) shifted by $\vec{R}$ and compare it to the transformation $\tilde{g}=(\tilde{\omega}, \tilde{\delta})$ written in terms of the new coordinate system $\tilde{x}$.

$$
\vec{x}+\vec{R}=\tilde{x}+\tilde{\delta}-\tilde{\omega} \times \tilde{x} \equiv[(\tilde{x}-\vec{R})+\vec{\delta}-\vec{\omega} \times(\tilde{x}-\vec{R})]+\vec{R}
$$

In order for $g$ and $\tilde{g}$ to act the same way, we must choose $\tilde{\omega}=\vec{\omega}$ and $\tilde{\delta}=\vec{\delta}-\vec{\omega} \times \vec{R}$. We note that not only does $(\vec{\omega}, \vec{\delta})$ transform as above, but in addition, due to the linearity of the transformation, $(\vec{\Omega}, \vec{\Delta})$ transforms the same way. As a result, we see that the derivatives of interest in (8) transform as

$$
\partial_{z} u_{i}=\left[\Delta_{i}-\epsilon_{i j k} \Omega_{j} \eta_{k}\right] / \delta_{3} \longrightarrow\left[\Delta_{i}+\epsilon_{i j k} \Omega_{j}\left(\eta_{k}-R_{k}\right)+\epsilon_{i j k} \Omega_{j} \vec{R}_{k}^{t r}\right] / \delta_{3},
$$

where $\vec{R}_{k}^{t r}$ is the projection of $\vec{R}$ onto the $\eta_{1}-\eta_{2}$ plane. Since changing the base point on each slab only requires components of $\vec{R}$ in that plane, we see that the last two terms in (23) cancel and the relevant derivatives are invariant with respect to the choice of the origin on the plate. Thus we are free to choose the most convenient origin for any calculation of interest.

\section{References}

[1] Marko J.F. and Siggia E.D., Macromolecules 24 (1994) 981-988. 
[2] Marko J.F. and Siggia E.D., Macromolecules 28 (1995) 8759-8770.

[3] Fain B., and Rudnick J., "Conformations of Linear DNA", preprint (cond-mat/9610126), (1996).

[4] Marko J.F., Europhys. Lett. 38 (1997) 183.

[5] Kamien R.D., Lubensky T.C., Nelson P, and O'Hern C. S., Europhys. Lett. 38 (1997) 237-242.

[6] Bouchiat C. and Mézard M., preprint (cond-mat/9706050), (1997).

[7] Smith S.B., Cui Y.J., and Bustamante C., Science 271 (1996) 795-799.

[8] Wang M.D., Yin H., Landick R., Gelles J., and Block S.M., Biophysical Journal 72 (1997) 1335-1346.

[9] Stasiak A. and Di Capua E., Nature 299 (1982) 185-186.

[10] Strick T.R., Allemand J.F., Bensimon D., Bensimon A., and Croquette V., Science 271 (1996) 1835-1837.

[11] Cluzel P. et. al., Science 271 (1996) 792-794.

[12] Kamien R.D., Lubensky T.C., Nelson P, and O'Hern C. S., "TwistStretch Elasticity of DNA", Proceedings of MRS Fall 1996 Meeting, Boston, MA.

[13] Moroz J.D. and Nelson P., preprint, (1997).

[14] The helical geometry of the DNA molecule alone cannot explain the twist-stretch coupling. Consider straightening out the helical sugarphosphate backbones without changing the length per phosphate $0.6 \mathrm{~nm}$ or the distance from the center of the molecule $0.9 \mathrm{~nm}$. Since each base pair step is $h=0.34 \mathrm{~nm}$, the circumferential length per step is $l_{c}=\sqrt{.6^{2}-.34^{2}} \mathrm{~nm}$. The corresponding twist angle per step is given by [15] $\theta=2 \arcsin \left(\left(l_{c} / 2\right) / .9 \mathrm{~nm}\right)=32^{\circ}$. An extension of the molecule by $\delta h / h=\epsilon$, yields an untwisting by an amount $\sigma=\delta \theta / \theta=\sigma_{\epsilon=0}-\epsilon / 2.0$. The twist response to stretch given by the coefficient of $\epsilon$ in $\sigma$ versus $\epsilon$ is much smaller than the coefficient $(\approx 7)$ found experimentally[10]. 
[15] Calladine C. R. and Drew H. R., Understanding DNA: the molecule and how it works, Academic, London (1992).

[16] Lavery R. and Sklenar H., Journal of Biomelecular Structure \& Dynamics 6 (1989) 655-667.

[17] Saenger W., Principles of Nucleic Acid Structure, Springer-Verlag, New York (1984).

[18] Krenk S., Journal of Applied Mechanics 50 (1983) 137-142. 


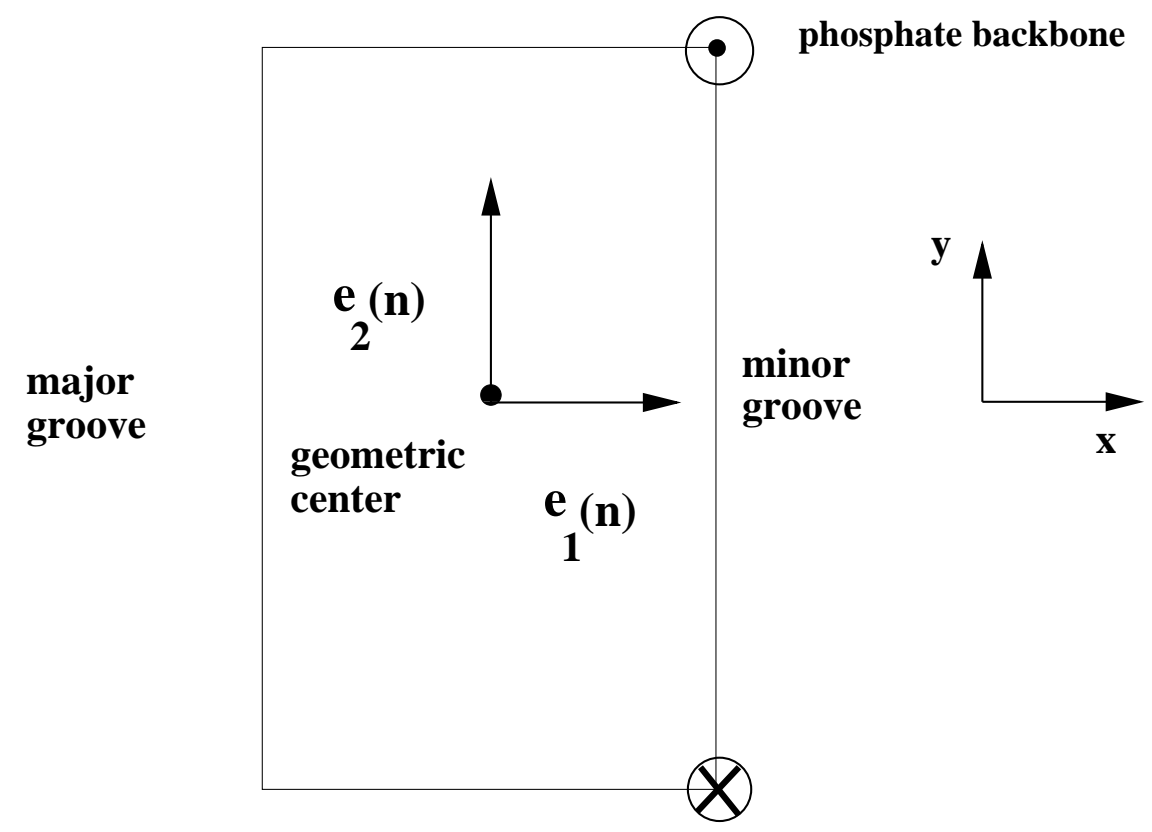

Figure 1: Schematic cross section of DNA taken perpendicular to the sugarphosphate backbones. $\mathbf{e}_{1}$ is perpendicular to the line connecting the two phosphate backbones and points from the geometric center of the plate toward the minor groove and $\mathbf{e}_{2}$ points from the geometric center toward the backbone running in the positive $\mathbf{e}_{3}$ direction. Each slice has a coordinate system $(x, y, z)$ with $\mathbf{e}_{1}$ as the $x$-axis, $\mathbf{e}_{2}$ as the $y$-axis, and $\mathbf{e}_{3}$ as the $z$-axis. Also, each slice is labeled by an integer $n$. 